\title{
Generalized Parton Distributions for Nucleon in the Impact Parameter Space
}

\author{
Neetika Sharma \\ Indian Institute of Science Education and Research Mohali, \\ S.A.S. Nagar, Mohali-140306, India \\ neetika@iisermohali.ac.in \\ Published 29 February 2016
}

\begin{abstract}
We investigate the generalized parton distributions (GPDs) for the proton and neutron in both momentum and position space in the soft-wall approach of anti-de Sitter/QCD model. The soft-wall model is based upon an action which incorporates confinement through the presence of a background dilaton field and the contribution of higher Fock states are holographically incorporated via studying the dynamics of 5D fermion fields of different scaling dimension. Our results are comparable with the other phenomenological models of the nucleon GPDs.
\end{abstract}

Keywords: Electromagnetic form factors; proton neutron; general properties of QCD.

PACS numbers: 13.40.Gp, 14.20.Dh, 12.38.Aw

\section{Introduction}

Generalized parton distributions (GPDs) are dynamical quantities of interest that have been studied extensively in the recent past. ${ }^{1,2}$ At the leading order, there are two helicity independent $\operatorname{GPDs}(H(x, \zeta, t)$ and $E(x, \zeta, t))$ for the valence quarks; where $x$ is longitudinal momentum fraction, $\zeta$ skewness parameter measuring the longitudinal momentum transferred, and $t=-Q^{2}$ is the square of total momentum transferred. GPDs combine and enlarge the information on ordinary parton distribution functions (PDFs) and the electromagnetic form factors (EFFs) into a single set of functions. ${ }^{3}$ They reduce to the PDFs in the forward limit and first moments are related to the EFFs. The impact parameter space dependent GPDs give the probability density of finding a quark in transverse impact parameter space. ${ }^{4}$

Deeply virtual compton scattering experiment is the main process to probe the information on GPDs. Various experiments such as $\mathrm{H} 1$ and ZEUS at DESY, ${ }^{5}$ COMPASS and COMPASS-II at CERN, ${ }^{6}$ Hall A and CLAS at Jefferson Lab ${ }^{7}$

This is an Open Access article published by World Scientific Publishing Company. It is distributed under the terms of the Creative Commons Attribution 3.0 (CC-BY) License. Further distribution of this work is permitted, provided the original work is properly cited. 
have measured the GPDs for valence quarks, sea quarks, and gluons. However, the numerical analysis is model dependent as it require a functional form of parameters $(x, \zeta, t) .{ }^{8,9}$ There are various alternative ways to extract information about the GPDs. One of the important procedure is based on direct parametrization of the PDF combined with the $Q^{2}$ dependence. It is difficult to incorporate the $Q^{2}$ dependence from the first principle, one can add the $Q^{2}$ dependence from the phenomenological description of the EFFs. ${ }^{10}$

Recently, light-front holography (LFH) has been developed as a promising technique to study the hadron structure. ${ }^{11}$ The formalism is developed by Brodsky and Teramond as a semiclassical approximation to QCD based on the AdS/CFT correspondence. ${ }^{12} \mathrm{LFH}$ procedures are based on the matching of matrix elements of dynamical obserables e.g. electromagnetic current matrix elements in AdS space with the corresponding expressions from light-front quantization in physical spacetime. AdS/QCD correspondence has been successfully applied to the general properties of mesons ${ }^{13-15}$ and baryons. ${ }^{16,17}$

One can also use the LFH principle to constrain the information on GPDs for valence quarks via matching the EFFs in the AdS models with the QCD sum rules that connect GPDs with form factors. This has lead to analytical expressions for GPDs in the soft-wall mode ${ }^{19}$ and hard-wall model. ${ }^{20}$ Recently, a variant of the holographic soft-wall model has been introduced by Gutsche et $_{\text {al. }}{ }^{21}$ in which the contribution of the higher Fock states has been incorporated via studying the dynamics of 5D fermion fields of different scaling dimension. We use the modified soft-wall model to investigated the GPDs for nucleon using the matching procedure. We further investigate the Fourier transform of GPDs to obtain information on the transverse impact parameter space.

\section{GPDs in the Soft-Wall Model of AdS/QCD}

The Dirac and Pauli form factors $F_{1}\left(q^{2}\right)$ and $F_{2}\left(q^{2}\right)$ for the spin $1 / 2$ particles are

$$
\left\langle P^{\prime}\left|J^{\mu}(0)\right| P\right\rangle=u\left(P^{\prime}\right)\left[F_{1}\left(q^{2}\right) \gamma^{\mu}+F_{2}\left(q^{2}\right) \frac{1}{2 M} \sigma^{\mu \alpha} q_{\alpha}\right] u(P),
$$

where $q^{2}=\left(P^{\prime}-P\right)^{2}$ is the momentum transferred, $M$ is the mass of nucleon, and $u(P)$ is the bound state spinor. The sum rules relating the proton EFFs with GPDs are given as

$$
\begin{aligned}
& F_{1}^{p}(t)=\int_{0}^{1} d x\left(\frac{2}{3} H^{u}(x, t)-\frac{1}{3} H^{d}(x, t)\right), \\
& F_{2}^{p}(t)=\int_{0}^{1} d x\left(\frac{2}{3} E^{u}(x, t)-\frac{1}{3} E^{d}(x, t)\right) .
\end{aligned}
$$

The sum rules for neutron can be obtained by the isospin symmetry. Also, we restrict our analysis to the contribution of the up and down quarks and antiquarks. 
Recently, Gutsche et al. ${ }^{21}$ have calculated the nucleon form factors using a modified soft-wall AdS/QCD model. The corresponding interaction Lagrangian which generates the nucleon form factors is expressed as

$$
\begin{aligned}
\mathcal{L}_{\mathrm{int}}(x, z)= & \sum_{i=+,-} \sum_{\tau} c_{\tau} \bar{\Psi}_{i, \tau}(x, z)\left(Q \Gamma^{M} V_{M}(x, z) \pm \frac{i}{4} \eta_{V}\left[\Gamma^{M}, \Gamma^{N}\right] V_{M N}(x, z)\right. \\
& \left. \pm g_{V} \tau_{3} \Gamma^{M} i \Gamma^{z} V_{M}(x, z)\right) \Psi_{i, \tau}(x, z)
\end{aligned}
$$

where $\Psi_{ \pm, \tau}(x, z)$ is fermonic field, $V_{M}(x, z)$ is vector field, and $V_{M N}=\partial_{M} V_{N}-$ $\partial_{N} V_{M}$ is the stress tensor of the vector field.

We perform the matching of EFFs in the AdS modes and the QCD sum rules which relate the EFFs to the GPDs. The expressions for up and down quark GPDs in modified soft-wall model of AdS/QCD are given as

$$
H^{q}\left(x, Q^{2}\right)=q(x) x^{Q^{2} / 4 \kappa^{2}}, \quad E^{q}\left(x, Q^{2}\right)=e^{q}(x) x^{Q^{2} / 4 \kappa^{2}} .
$$

where the quark distribution functions $q(x)$ and $e^{q}(x)$ are

$$
q(x)=\alpha_{1}^{q} \gamma_{1}(x)+\alpha_{2}^{q} \gamma_{2}(x)+\alpha_{3}^{q} \gamma_{3}(x), \quad e^{q}(x)=\alpha_{3}^{q} \gamma_{4}(x) .
$$

The flavor coupling parameters $\alpha_{i}^{q}$ and $\gamma_{i}(x)$ are expressed as

$$
\begin{aligned}
& \alpha_{1}^{u}=2, \alpha_{2}^{u}=g_{V}, \alpha_{3}^{u}=2 \eta_{V}^{p}+\eta_{V}^{n}, \quad \alpha_{1}^{d}=1, \alpha_{2}^{d}=-g_{V}, \alpha_{3}^{d}=\eta_{V}^{p}+2 \eta_{V}^{n}, \\
& \gamma_{1}(x)=-\frac{1}{2} \sum_{\tau} c_{\tau}(1-2 \tau+x \tau)(1-x)^{\tau-2}, \gamma_{2}(x)=\frac{1}{2} \sum_{\tau} c_{\tau}(1-x \tau)(1-x)^{\tau-2} \\
& \gamma_{3}(x)=\sum_{\tau} c_{\tau}\left(1-3 x \tau+x^{2} \tau(1+\tau)\right)(1-x)^{\tau-2}, \\
& \gamma_{4}(x)=\frac{2 M_{n}}{\kappa} \sum_{\tau} c_{\tau} \tau \sqrt{\tau-1}(1-x)^{\tau-1} .
\end{aligned}
$$

We consider the contribution of three leading twist $\tau=3,4,5$ terms. In Fig. 1(a)-1(b), we have presented the behavior of spin conserving GPD $H\left(x, Q^{2}\right)$ for the up and down quarks. In Fig. $1(\mathrm{c})-1(\mathrm{~d})$, we have plotted the spin changing GPD $E\left(x, Q^{2}\right)$ for the up and down quarks. The qualitative behavior of GPDs are same for both the quarks. It is interesting to know that the profile function increase with $x$ to maximum value and then decrease further as $x$ increase.

The higher order moments in $x$ for the valence GPDs are defined as

$$
\begin{aligned}
& H_{n}^{q}\left(Q^{2}\right)=\int_{0}^{1} \mathrm{~d} x x^{n-1} H^{q}\left(x, Q^{2}\right), \\
& E_{n}^{q}\left(Q^{2}\right)=\int_{0}^{1} \mathrm{~d} x x^{n-1} E^{q}\left(x, Q^{2}\right) .
\end{aligned}
$$

The first moment of GPDs give the EFFs and the second moment correspond to gravitational form factors. The higher order moments correspond to the form factors 
(a)

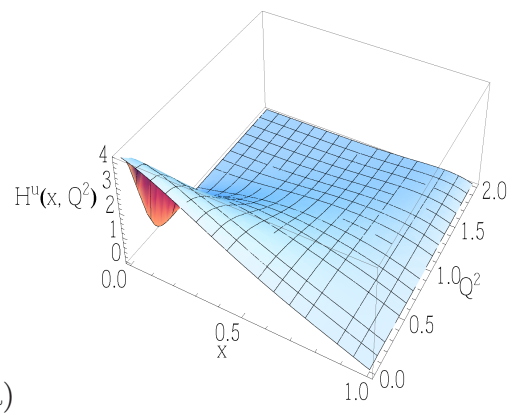

(c)

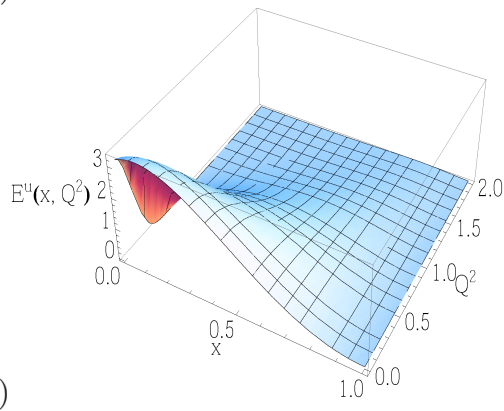

(b)

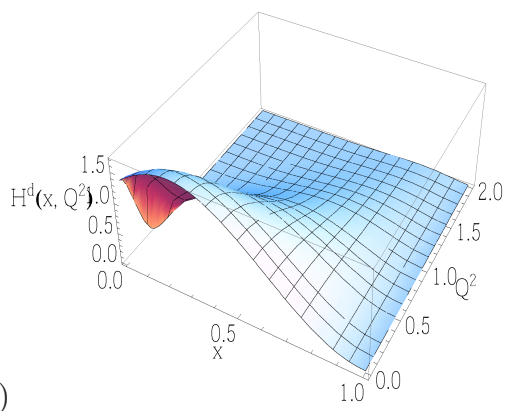

(d)

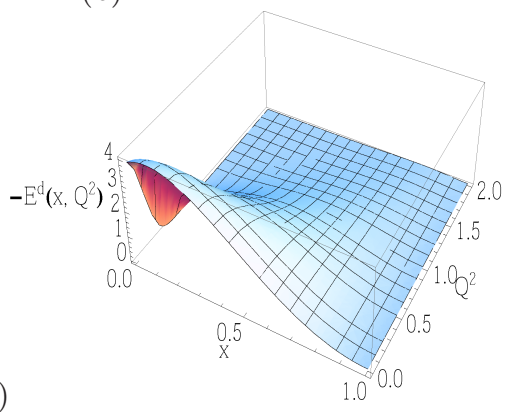

Fig. 1. Plots of the generalized parton distributions (a) $H^{u}\left(x, Q^{2}\right)$, (b) $H^{d}\left(x, Q^{2}\right)$; (c) $E^{u}\left(x, Q^{2}\right)$ and (d) $-E^{d}\left(x, Q^{2}\right)$.

of higher-twist operators. In Figs. $2(\mathrm{a})-2(\mathrm{~d})$, we have plotted the behavior of first three moment of GPDs $Q^{2} H_{n}^{u / d}\left(Q^{2}\right)$ and $Q^{2} E_{n}^{u / d}\left(Q^{2}\right)$ with momentum $Q^{2}$ for up and down quarks. We observe that the behavior of GPD moments with $Q^{2}$ is same as the behavior of profile functions with momentum fraction $x$ and the variation become slower as index $n$ increases.

\section{GPDs in Impact Parameter Space}

GPDs in the impact parameter space are related to the Fourier transform of GPDs in the momentum space. The impact parameter GPDs give the probability density for finding a quark with a longitudinal momentum fraction $(x)$ and transverse position $\left(b_{\perp}\right)$ for the unpolarized nucleon. The GPDs in transverse impact parameter space ${ }^{4}$

$$
\begin{aligned}
q\left(x, b_{\perp}\right) & =\int \frac{\mathrm{d}^{2} q_{\perp}}{(2 \pi)^{2}} e^{\iota b_{\perp} \cdot q_{\perp}} H\left(x, q^{2}\right), \\
e^{q}\left(x, b_{\perp}\right) & =\int \frac{\mathrm{d}^{2} q_{\perp}}{(2 \pi)^{2}} e^{\iota b_{\perp} \cdot q_{\perp}} E\left(x, q^{2}\right) .
\end{aligned}
$$

In Figs. 3(a)-3(b), we have plotted the behavior of impact parameter GPDs $u(x, b)$ and $d(x, b)$ and in Figs. 3(c)-(d), we have plotted the GPDs $e^{u}(x, b)$ and $e^{d}(x, b)$ for the up and down quarks. The qualitative behavior of both the GPDs $q(x, b)$ and 


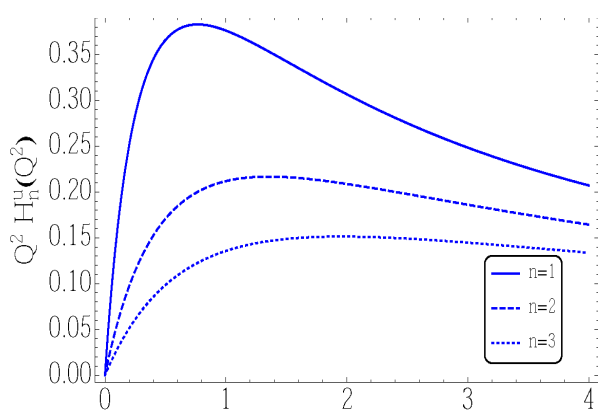

(a)

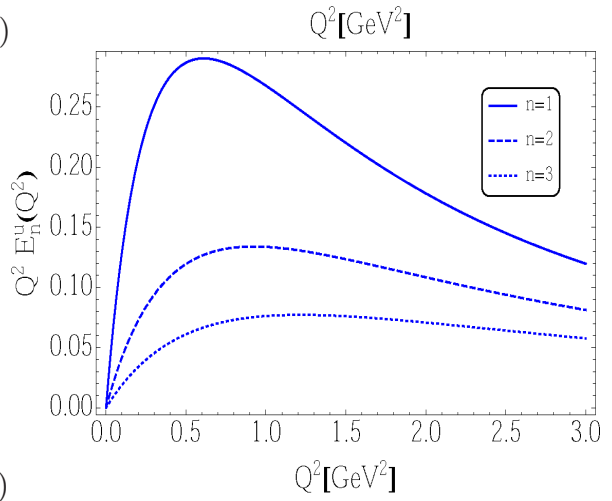

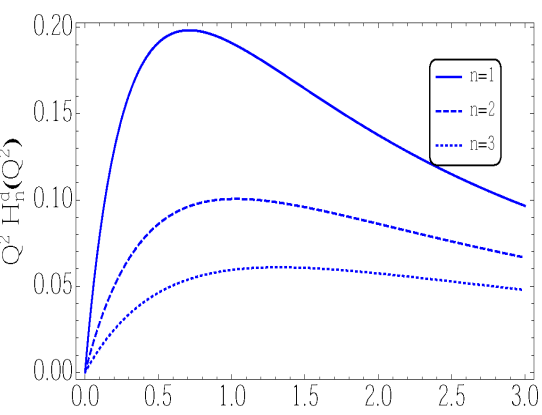

(b)

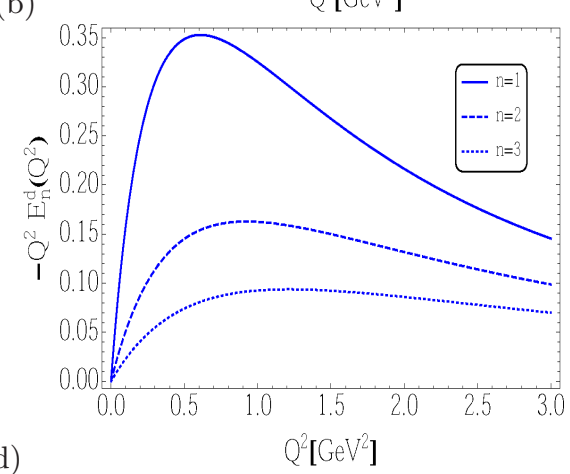

Fig. 2. Plots of first three moments of generalized parton distribution (a) $Q^{2} H_{n}^{u}\left(x, Q^{2}\right)$ $Q^{2} H_{n}^{d}\left(x, Q^{2}\right)$; (c) $Q^{2} E_{n}^{u}\left(x, Q^{2}\right)$ (d) $Q^{2} E_{n}\left(x, Q^{2}\right)$ vs $Q^{2}$ in the holographic soft-wall model

$e^{q}(x, b)$ is same for both up and down quarks. The peak of GPDs shifts towards a lower value of $x$ as $b$ increase, therefore the transverse profile is peaked at $b=0$ and decrease further. We observe that for the small values of $b$, the magnitude of GPD $q(x, b)$ is larger for up than down quark, whereas the magnitude of the GPD $e^{q}(x, b)$ is almost same for both quarks.

\section{Summary and Conclusion}

In this work, we have numerically studied the helicity independent GPDs for nucleon. We have used a variant of soft-wall model of AdS/QCD in which the contribution of higher fock states is holographically incorporated in the fermion field. We have used the light-front holography principle to match the nucleon EFFs in AdS modes with the sum rules in QCD. We have presented the explicit results for the moments of GPDs in the momentum space. Further, we investigated the variation of GPDs in the impact parameter space. Though in the AdS/QCD we have considered only valence quarks GPDs, it is interesting to observe that their behaviors are quite similar and in agreement with the other phenomenological models in both momentum space and impact parameter space. 
(a)

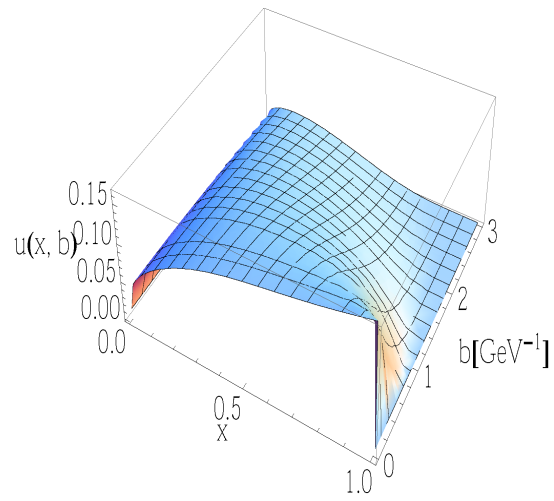

(c)

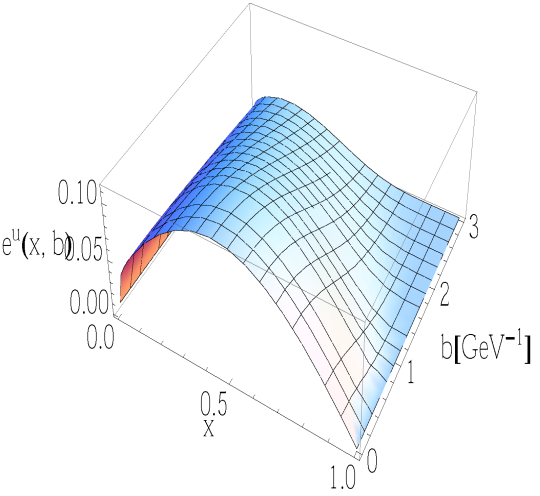

(b)

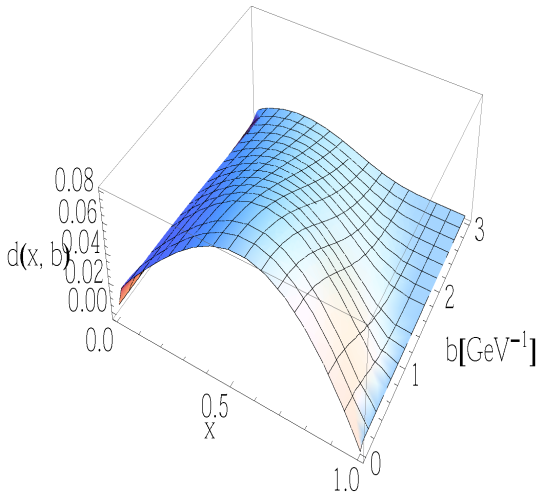

(d)

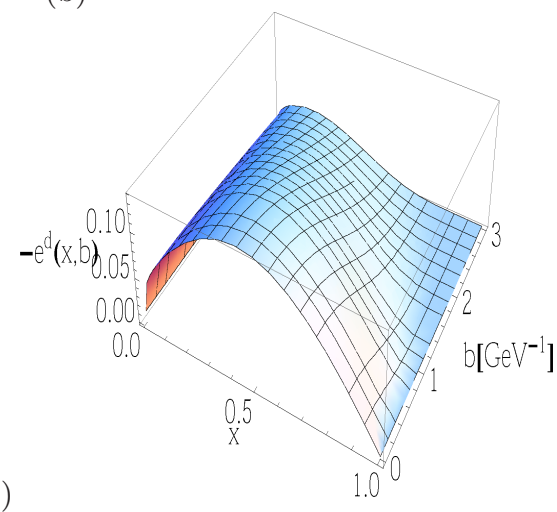

Fig. 3. (Color online) Plots of the impact parameter dependent GPDs (a) $u\left(x, b_{\perp}\right)$; (b) $d\left(x, b_{\perp}\right)$; (c) $e^{u}\left(x, b_{\perp}\right)$, and $(\mathrm{d})-e^{d}\left(x, b_{\perp}\right)$ in the holographic soft-wall model.

\section{Acknowledgments}

This work is supported by the Department of Science and Technology, Government of India, Grant No. SR/FTP/PS-057/2012.

\section{References}

1. K. Goeke, M.V. Polyakov, and M. Vanderhaeghen, Annu. Rev. Nucl. Part. Sci. 54, 413 (2004).

2. A.V. Belitsky and A.V. Radyushkin, Phys. Rep. 418, 1 (2005).

3. X. Ji, Phys. Rev. Lett. 78, 610 (1997).

4. M. Burkardt, Phys. Rev. D 62, 071503 (2000); Erratum 66, 119903E (2002).

5. S. Chekanov et al. (ZEUS Collaboration), J. High Energy Phys. 0905, 108 (2009).

6. C. Adolph et al. (COMPASS Collaboration), Phys. Lett. B 717, 383 (2012).

7. F. Girod et al. (CLAS Collaboration), Phys. Rev. Lett. 100, 162002 (2008).

8. J.J. Kelly, Phys. Rev. C 70, 068202 (2004).

9. A.D. Martin, W.J. Stirling, R.S. Thorne, and G. Watt, Eur. Phys. J. C 63, 189 (2009).

10. O. V. Selyugin, Phys. Rev D 89, 093007 (2014)

11. S.J. Brodsky and G.F. de Teramond, Phys. Rev. Lett. 96, 201601 (2006).

12. G.F. de Teramond and S.J. Brodsky, Phys. Rev. Lett. 102, 081601 (2009). 
13. H.J. Kwee and R.F. Lebed, J. High Energy Phys. 01, 27 (2008).

14. S.J. Brodsky and G.F. de Teramond, Phys. Rev. D 77, 056007 (2008)

15. J.R. Forshaw and R. Sandapen, Phys. Rev. Lett. 109, 081601 (2012).

16. S.J. Brodsky, F.G. Cao and G.F. de Teramond, Phys. Rev. D 84, 033001 (2011).

17. Z. Abidin and C.E. Carlson, Phys. Rev. D 79, 115003 (2009).

18. S.J. Brodsky and G.F. de Teramond, arXiv:1203.4025.

19. D. Chakrabarti and C. Mondal, Phys. Rev. D 88, 073006 (2013).

20. A. Vega, I. Schmidt, T. Gutsche, V.E. Lyubovitskij, Phys. Rev. D 85, 096004 (2012).

21. T. Gutsche, V.E. Lyubovitskij, I. Schmidt, and A. Vega, Phys. Rev. D 86, 036007 (2012); N. Sharma, Phys. Rev. D 90, 095024 (2014). 